\title{
Active Matter Alters the Growth Dynamics of Coffee Rings
}

\author{
Agnese Callegari, Tugba Andac, Pascal Weigmann \\ Soft Matter Lab, Department of Physics, Bilkent University, Ankara 06800, Turkey \\ callegari@fen.bilkent.edu.tr \\ Sabareesh K. P. Velu \\ Soft Matter Lab, Department of Physics, Bilkent University, Ankara 06800, Turkey \\ University of Information Science and Technology "St.Paul the Apostle", 6000 - Ohrid, R. Macedonia \\ Ercag Pince \\ Soft Matter Lab, Department of Physics, Bilkent University, Ankara 06800, Turkey \\ Giorgio Volpe \\ Department of Chemistry, University College London, 20 Gordon Street, London WC1H 0AJ, United Kingdom

\section{Giovanni Volpe} \\ Göteborgs Universitet, Institutionen för Fysik, 41296 Göteborg, Sweden \\ Soft Matter Lab, Department of Physics, Bilkent University, Ankara 06800, Turkey
}

\begin{abstract}
We show that bacterial mobility starts playing a major role in determining the growth dynamics of the edge of drying droplets, as the droplet evaporation rate slows down. ( $) 2018$ The Author(s) OCIS codes: (000.2190) Experimental Physics; (000.6590) Statistical Mechanics; (180.1790) Confocal microscopy; (350.4990) Particles
\end{abstract}

How particles are deposited at the edge of evaporating droplets, i.e. the coffee ring effect [1-5], plays a crucial role in phenomena as diverse as thin-film deposition, self-assembly, and biofilm formation. Recently, microorganisms have been shown to passively exploit and alter these deposition dynamics to increase their survival chances under harshening conditions [6-10]. Here, we show that, as the droplet evaporation rate slows down, bacterial mobility starts playing a major role in determining the growth dynamics of the edge of drying droplets. Such motility-induced dynamics can influence several biophysical phenomena, from the formation of biofilms to the spreading of pathogens in humid environments and on surfaces subject to periodic drying. Analogous dynamics in other active matter systems can be exploited for technological applications in printing, coating, and self-assembly, where the standard coffee-ring effect is often a nuisance.
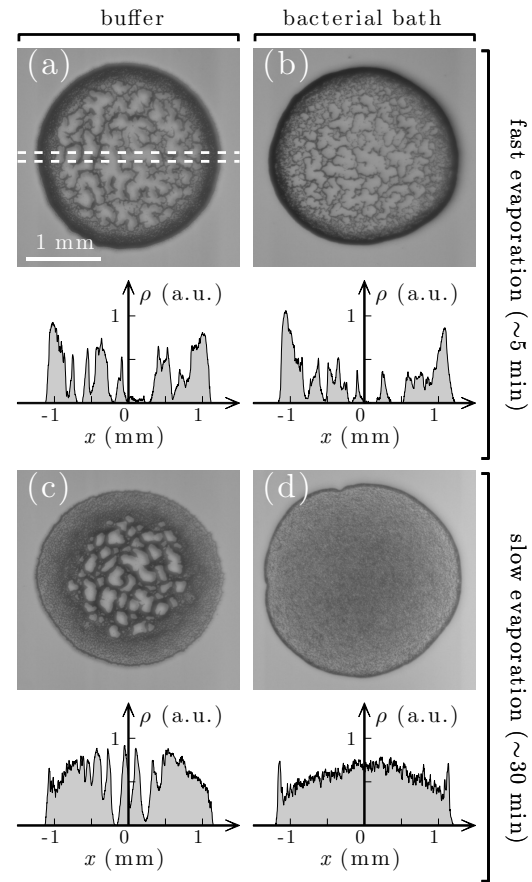

Figure 1: Stain at the end of evaporation with and without bacteria. Stain left behind by a droplet (a,b) after fast evaporation ( $\sim 5$ min) 
and (c,d) after slow evaporation ( $\sim 30 \mathrm{~min})$. The droplet is made of a buffer solution containing colloidal particles (polystyrene microsphere, diameter $2 \mathrm{R}=3.00 \pm 0.07 \mu \mathrm{m})(\mathrm{a}, \mathrm{c})$ without and $(\mathrm{b}, \mathrm{d})$ with motile bacteria (E. Coli). The plot below each panel shows the optical density of the deposit along one droplet's diameter (dashed line in (a)) as calculated from the image inverted gray scale. (a,b) For fast evaporation, both stain share similar features. (c,d) For slow evaporation, the stain of the droplet containing bacteria features higher uniformity than all other cases. From [11]
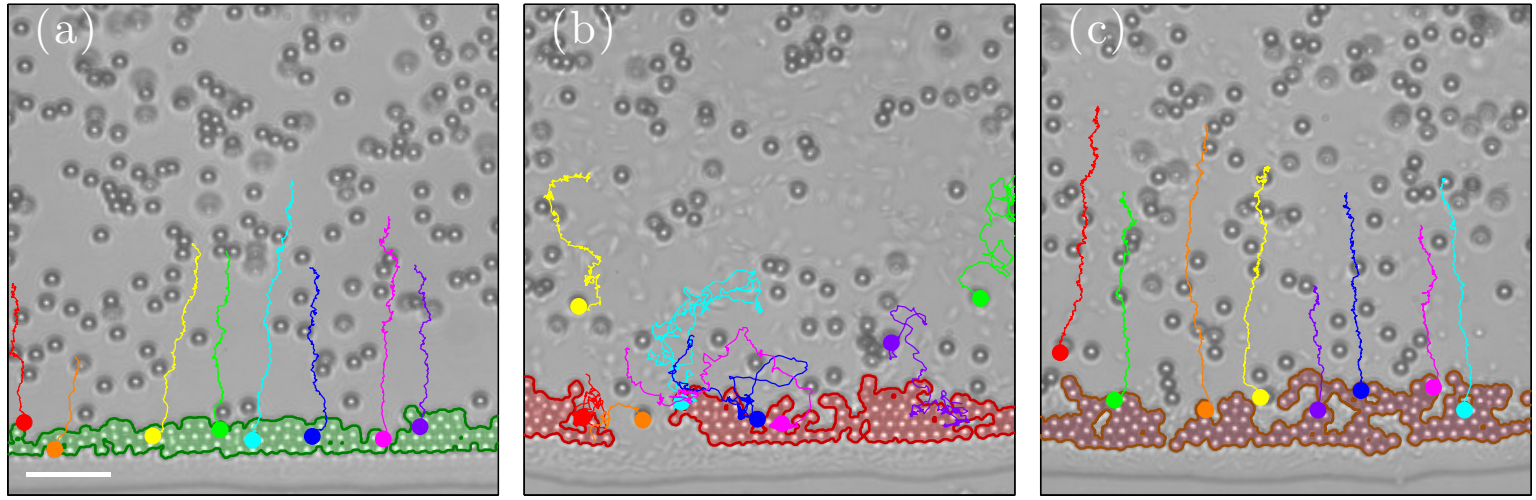

Figure 2: Growth dynamics at the edge of drying droplets. Edge of a drying droplet of motility buffer containing (a) only colloidal particles, (b) colloidal particles and motile bacteria, and (c) colloidal particles and non-motile bacteria. The shaded area represents the already formed border, and the colid lines show the trajectories of some particles recorded over the preceding $60 \mathrm{~s}$. In (b), because of the presence of motile bacteria, the particle trajectories are more complex and also feature events where a particle escapes the boundary after having reached it. The scalebar corresponds to $20 \mu \mathrm{m}$. From [11]

\section{References}

[1] R. Brown, “Additional remarks on active molecules," Phil. Mag., 6, 161-166 (1829)

[2] R. E. Goldstein, "Coffee stains, cell receptors, and time crystals: Lessons from the old literature," Phys. Today, 71, in press (2018)

[3] R. D. Deegan, O. Bakajin, T. F. Dupont, G. Huber, S. R. Nagel, and T. A. Witten. "Capillary flow as the cause of ring stains from dried liquid drops." Nature, 389, 827-829 (1997).

[4] R. D. Deegan, O. Bakajin, T. F. Dupont, G. Huber, S. R. Nagel, and T. A. Witten, "Contact line deposits in an evaporating drop," Phys. Rev. E, 62, 756-765 (2000)

[5] R. D. Deegan, "Pattern formation in drying drops," Phys. Rev. E, 61, 475-485 (2000)

[6] W. Sempels, R. De Dier, H. Mizuno, J. Hofkens, and J. Vermant, "Auto-production of biosurfactants reverses the coffee ring effect in a bacterial system," Nat. Commun., 4, 1757 (2013)

[7] D. Yanni, A. Kalziqi, J. Thomas, S. L. Ng, S. Vivek, W. C. Ratcliff, B. K. Hammer, and P. J. Yunker, "Life in the coffee-ring: How evaporation-driven density gradients dictate the outcome of inter-bacterial competition," arXiv, 1707.03472 (2017)

[8] G. A. Turnbull, J. A. W. Morgan, J. M. Whipps, and J. R. Saunders, "The role of bacterial motility in the sur- vival and spread of Pseudomonas fluorescens in soil and in the attachment and colonisation of wheat roots," FEMS Microbiol. Ecol., 36, 21-31 (2001)

[9] C. Matz and K. Jürgens, "High motility reduces grazing mortality of planktonic bacteria," Appl. Environmental Microbiol., 71, 921-929 (2005)

[10] M. Hennes, J. Tailleur, G. Charron, and A. Daerr, "Active depinning of bacterial droplets: The collective surfing of Bacillus Subtilis," Proc. Natl. Acad. Sci. U.S.A., 114, 5958-5963 (2017)

[11] T. Andac, P. Weigmann, S. K. P. Velu, E. Pince, A. Callegari, G. Volpe, G. Volpe, “Active Matter Alters the Growth Dynamics of Coffee Rings," arXiv:1803.02619 [cond-mat.soft] (2018) 